\title{
Designing a Building envelope using parametric and algorithmic processes
}

\author{
Natália Queiroz \\ Universidade Federal de Pernambuco, Brazil \\ nataliaqueiroz.arq@gmail.com \\ - Ney Dantas \\ Universidade Federal de Pernambuco, Brazil \\ ney.dantas@gmail.com
}

\author{
- Carlos Nome \\ Universidade Federal da Paraiba, Brazil \\ carlos.nome@gmail.com \\ - Carlos Vaz \\ Universidade Federal de Pernambuco, Brazil \\ cev00@gmail.com
}

\begin{abstract}
This article presents a case study on a vertical building envelope design considering Recife's climatic conditions. It consists of the utilization of parametric and algorithmic processes to distribute sun shading devices. The study uses computational tools such as Grasshopper and the DIVA for Grasshopper. The latter is a thermal energy simulation tool that uses the Energy Plus 7.0 and Daysim for calculations. The model uses incident solar radiation simulation on the envelope as basis for distribution of shading devices. The result is a parametric model that automatically responds to urban and climatic characteristics, through simulations integrated to the 3D model.
\end{abstract}

Keywords: Shading Devices, Parameterization, Grasshopper, DIVA

\section{Introduction}

There are many tools available that aid building envelope design and the consideration of its interaction with climactic conditions. Nonetheless, the integration of such tools to the design process is, more than often, problematic, especially in the schematic design stage. In practice there is a tendency to delay the incorporation of performance criteria to later design stages (Turrin et al., 2011; Lima, 2012).

According to Motta(1999), once identified, given parts of a design processes can be parameterized and reused in different design scenarios. The use of parametric and algorithmic processes can potentially automate parts of the design process and integrate multidisciplinary considerations in a single model at any given point in the design process. Thus, such processes can be incorporated in early design stages, where they are the most advantageous (Eastman 2008).

This short paper presents a case study on the use of parametric and algorithmic tools for the design of a vertical building's envelope in a hot and humid climate. The parametric model is established using graphic scripting, incident radiation computational simulation, integrated to the 3D modeling process. The model was developed, within a single interface, to automate the simulation and associate the shading device distribution to the simulation results.

Parametric and algorithmic designs, as well as, bioclimatic strategies for Recife are crucial aspects to this study. The first, presents the conceptual basis for the modeling processes and its association to performance criteria. The latter, reviews the main bioclimatic design strategies for Recife's climate and, consequent recommendations for envelope design.

Leach (2014) describes Parametric Design as the utilization of parametric modeling software. Such software allows the association of dimensional parameters and geometry, consequently allowing for model adjustments and variations without rework. Algorithmic design refers to the use of scripting languages that allow the user to design directly through code manipulation. This approach contributes to the reduction of limitations of single interface applications as well as the need for multiple software integration. The association of parametric and algorithmic processes contributes to the reduction of restrictions regarding the integration of analysis, modeling and fabrication processes (Oxman, 2010).

Woodbury (2010) indicates six skills necessary for algorithmic and parametric designers: workflow conception; ability to divide and connect logical parts; ability to name such parts; abstract thinking; mathematical thinking; and finally, ability to think algorithmically.

Graphic scripting languages allow programming through the connection of graphic elements instead of textual coding. This generates a visual model of data flow that, associated to 3D modeling tools, permits designers to program and execute integrated processes relevant to any given design effort. The use of visual scripting allows for similar results to the ones 
attained in algorithmic environments without the need for textual coding (Celani and Vaz, 2012)

The comprehension of the climatic context of a building design is a fundamental element in the incorporation of bioclimatic strategies in parametric processes. The context in this case is the city of Recife, Brazil, located in the coast at latitude of $08^{\circ} 08^{\prime} \mathrm{S}$ and longitude of $34^{\circ} 55^{\prime} \mathrm{W}$. It has a tropical hot and humid climate, with average temperatures ranging from $24^{\circ} \mathrm{C}$ to $28^{\circ} \mathrm{C}$ during the summer and from $22^{\circ} \mathrm{C}$ to $24^{\circ} \mathrm{C}$ during the winter months. Based on the adaptive model proposed by ASHARE standard 55 of 2010, Recifes's climate proportionate $59 \%$ of the hours in thermal comfort and $41 \%$ in discomfort . According to Givoni's Psichrometric Chart the main design strategies for Recife's climate are shading and ventilation respectively. Where, shading is a bioclimatic strategy recommended for $99,9 \%$ of the year and, ventilation for $67,9 \%$ of the year.

\section{Methodology}

The method consists of a case study on the use of parametric and algorithmic tools for the distribution of shading devices, with different geometric attributes, on the envelope of a vertical building. This is achieved through the use of a data grid generated by simulating incident solar radiation on the building's envelope. Results are discussed based on the analysis of the incident radiation and resulting application. Three development stages were adopted:

Definition of shading devices - establishes different attributes based on Recife's solar geometry and climate. This stage uses sunpath charts as well as the city's reference year climatic data to establish Vertical Shading Angles (VSA) and Horizontal Shading Angles (HSA).

Development of the parametric and algorithmic model - uses Grasshopper for Rhino 3D and includes incident solar radiation simulation in Recife as parameter for shading device distribution. The simulation is performed using DIVA, a pligin for Grasshopper that uses EnergyPlus 7.0 and Daysim.

Analysis of the results - Uses computer simulation to evaluate shading device distribution.

\section{Results}

\section{Definition of the shading devices}

The site used in the case study has an area of $2368 \mathrm{~m}^{2}$ and is located in the metropolitan region of Recife, and surrounded by vertical buildings. According to the climate reading the design premise is the development of an entirely shaded and permeable building envelope. This envelope should be reflective and parametrically distributed.

Recife's Sunpath chart is almost symmetrical due to its proximity to the Equator Line. The highest indices of incident radiation occurs at solar altitudes between $0^{\circ}$ and $60^{\circ}$, and peaks at altitudes between $0^{\circ}$ and $30^{\circ}$ (fig.1).

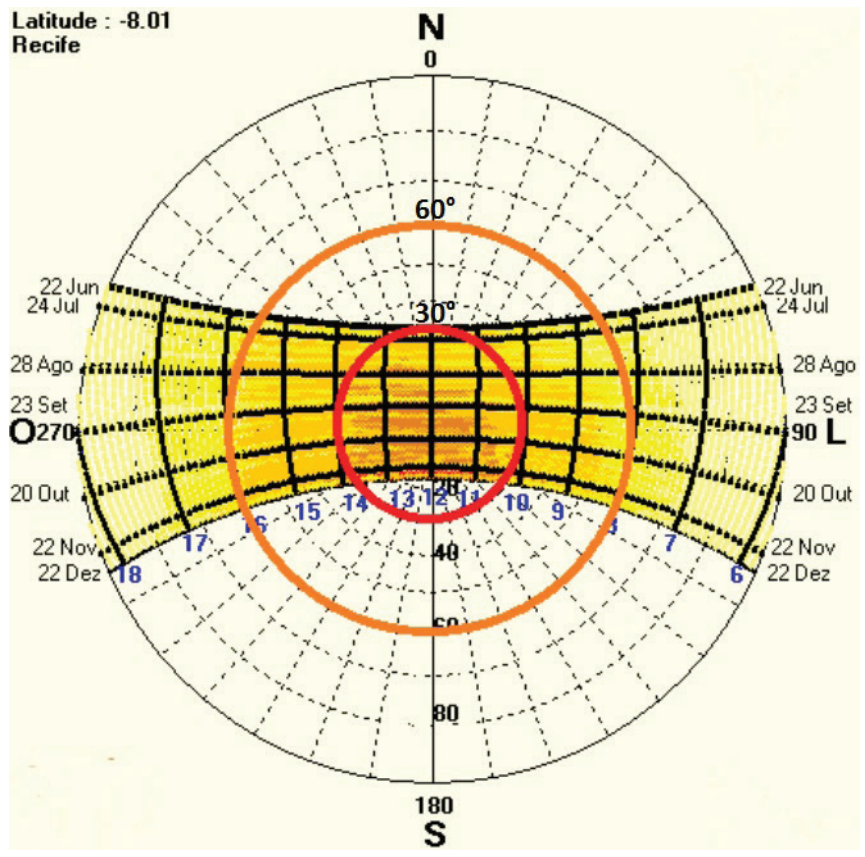

Figure 1: Recife's sunpath chart with global horizontal radiation scale. The red circle indicate values that represent a $30^{\circ}$ angle and the orange circle a $60^{\circ}$ angle. Within those circles are the highest incidences of radiation.

The model uses modular shading devices. Three devices were developed in response to Recife's solar geometry. One with a shading angle of $30^{\circ}$ for less demanding shading needs. A second one was developed, with a shading angle of $45^{\circ}$, for intermediate shading demands. And finally, a third shading device was developed, for areas that have high levels of incident radiation, with a shading angle of $60^{\circ}$. All of the devices have a horizontal shading angle in response to solar azimuth variations throughout the year (fig. 2).

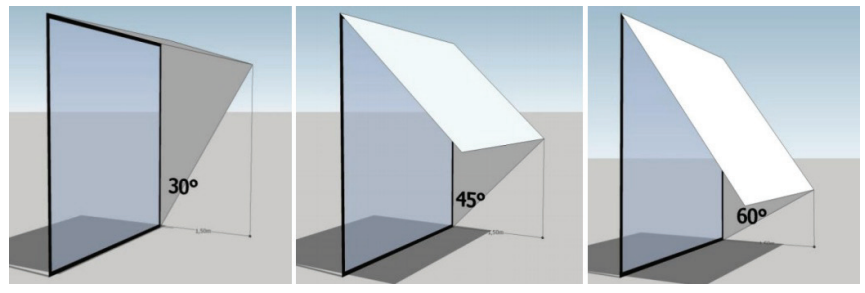

Figure 2: Three distinct modular shading devices (simplified model).

\section{Construction of the parametric/algorithmic model}

The construction of the parametric model used the plugin Grasshopper for Rhinoceros 3D. The Grasshopper model considered three inputs: 1 . Simplified site surroundings model; 2. the adopted architectural parti; and, 3. Models of the three types of shading devices. Both can be edited in the 3D software or modeled using graphic scripting.

The model includes the climactic file, incident radiation simulation (using the plugin DIVA) and, shading device 
distribution. The adopted approach allows for modification of dimensional parameter and evaluation of the resulting variations with ease. These dimensional parameters are associated to the distribution and quantity of shading devices as well as simulation grid controls. Once new parameters are established the model is updated in real time unveiling the new scenario. Figure 3 illustrates the behavior of the developed model.

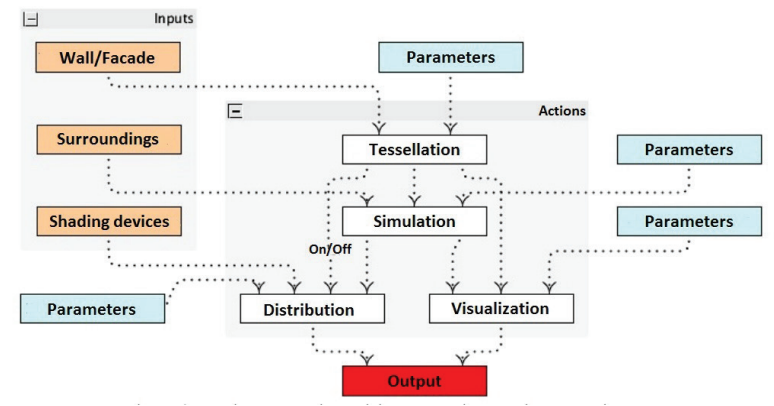

Figure 3: Proposed functioning of the parametric model generated in Grasshopper.

Simplified simulation options are available in DIVA for Grasshopper, these are important for considerations in early design stages. The parametric model uses the Daysym based lighting simulation module. The EPW climatic data file for Recife was incorporated. The Solar Radiation Nodes for the entire year option was adopted. This option generates a data list in $\mathrm{Kwh} /\left(\mathrm{m}^{2 *}\right.$ year) calculated from a tessellated mesh applied to the architectural parti.

Associating the architectural parti as entry data, allows for the visualization, in the model, of the surface pattern of incident radiation. In turn, this also allows for a better understanding of the performance of different geometrical explorations at early stages of the design process. Through such approach, as the designer makes decisions about the geometry in the 3D editing interface it is informed in the model about incident radiation in a configurable iso values data grid.

Values generated through the simulation, in Kwh/ $\left(\mathrm{m}^{2 *}\right.$ year),are used to distribute shading devices as specified by the modeler. Distribution parameters are editable and, can be used in combination with other parameters to test diverse scenarios. The model produces outputs that can assist with geometrical information take offs, such as: quantities of each type of shading devices.

\section{Analysis of the Results}

After the definition of the parametric model the simplified 3D model of the site surrounding was added, as well asgeometrical information on the building envelope from the architectural parti. In order to achieve improved understanding of the proposed envelope's performance the option of detaching the simulation from the geometry was added to the parametric model (Fig.4).

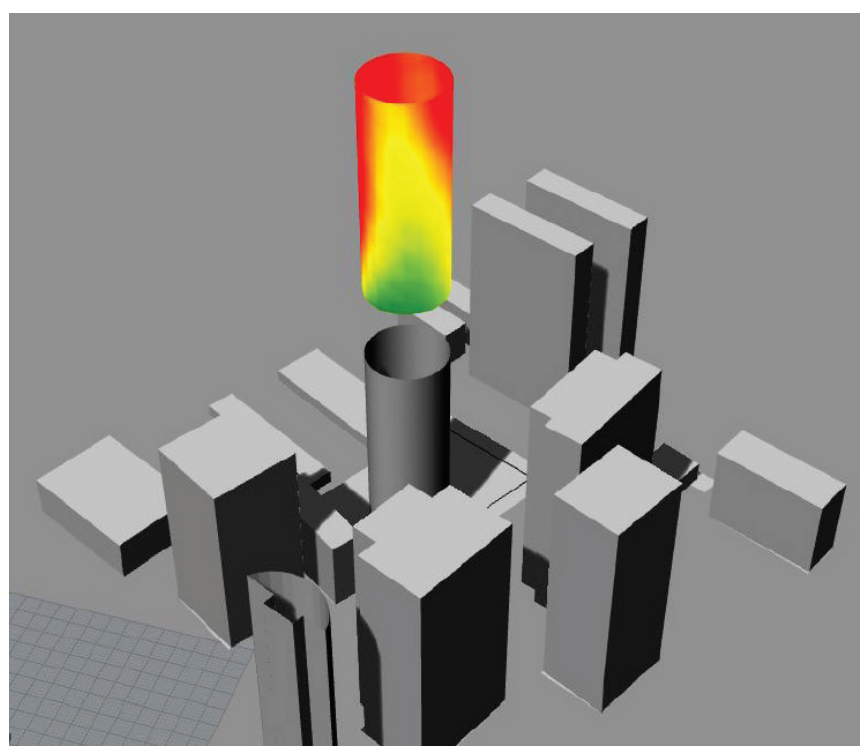

Figure 4: Generated model displaying simulation of incident radiation on the building envelope while architectural parti is under development.

Simulation results show great variation of incident radiation on the building envelope. Surrounding buildings have a significant influence on the results, to the extent that they define a shaded area at the base of the building envelope that has no need for shading devices. Distribution of shading devices follows the data grid resulting from the simulation as the parametric model's distribution model is turned on.

Three alternative combinations of shading devices and distribution patterns were tested in approximately 10 minutes. These alternatives varied shading device shading angles and, their distribution density on the building envelope. The results show the alternative understood as having the best performance potential.

The parametric application of shading devices responded to the proposed geometric and site surroundings scenario and, distributed them in accordance to the incident radiation simulation results. Naturally, the reduction of incident radiation on the building envelope was variable. Thus, after the distribution of shading devices incident radiation values on the building envelope became almost uniform (Fig. 5). 


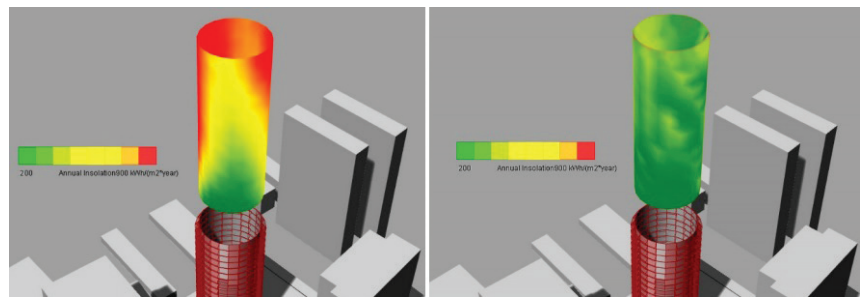

Figure 5: Color scale displaying incident radiation quantities on the building envelope prior to shading device distribution (left); after the parametric distribution of shading devices (right).

Shading the entire building envelope in climates such as Recife's climate reduces the influence of solar radiation on the building. This is positive in terms of thermal and energetic building performance, as well as, the reduction of the impact of the building on the thermal conditioning of its immediate surrounding (heat island effect). As a result of such explorations, there is an opportunity for abundant building openings, potential for improved lighting comfort through natural lighting strategies and, options for permeability and natural ventilation strategies. Although modular, the shading devices had a nonlinear distribution resulting in an organic appearance throughout the building envelope (Fig. 6).

The solution with a parametric approach extrapolates the restriction of traditional solutions and its characterization is directly related to the simulation results. This approach indicates potential uses for simulation and database structures in early design stages. Examples are: aid for building geometry definitions; performance optimization processes; quantity take offs; and, alternative solution testing while reducing rework.

The established parametric model can be used in future simulations. As permitted by visual scripting languages, this model can incorporate other parametric models as input data or, become a part of a more complex algorithmic model. It could also become a part of a genetic algorithmic model with integrated multi simulation models, which include other

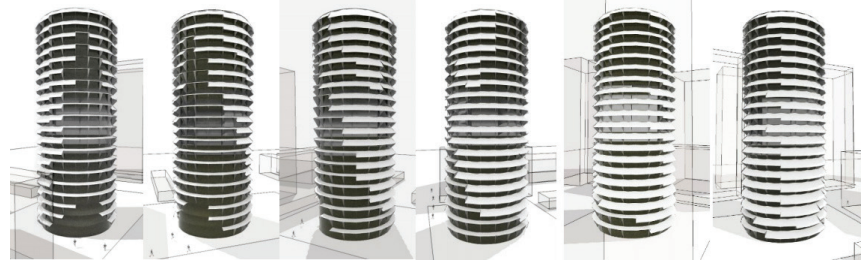

types of simulations, such as lighting and structural.

Figure 6: Formal results for preliminary study in sequential points of views.

\section{Conclusion}

The presented case study explores, through a parametric and algorithmic approach, the responsive design of a building envelope incorporating energy efficiency and bioclimatic considerations. It achieves a solution with potential to reduce ill effects of the building on the immediate urban thermal conditioning. Asproposed, the application integrates in a single interface interoperable geometric modeling, simulation, and graphic scripting tools. The study characterizes the ease with which information can be extracted from the model, in short time intervals, and the potential for immediate repercussion on building design decisions.

In terms of design process, the case study emulates possible considerations necessary for early design stages, such as the preliminary design stage. Traditional processes incorporate building envelope performance considerations at later stages. Understanding the effects of building geometry and its envelope in terms of energy efficiency can have a deep impact on the entire building lifecycle, especially during the building commissioning and operation.

Regarding exploration of alternative solutions to a given architectural parti, parametric and algorithmic processes offer unsurpassed opportunities when compared to traditional processes. Within a given geometric solution, designers can alter parameters to test adjustments in almost real time. Furthermore, there is the opportunity to test multiple geometries in the same model. Finally the potential reuse of the parametric and algorithmic model in other design scenarios elevates exponentially the potential benefits of such approaches.

The incorporation of evaluative cycles in the design process, associated to responsive design principles, allows more freedom for adjustments and combination of variations in response to a given design problem. This combination expands the users control over the limits imposed by traditional modeling and simulation tools, contributing to improve designer understanding of performance patterns and proposed solutions.

Algorithmic processes can contribute to improve design processes, as they reduce the influence of limitations of traditional modeling tools by allowing the designer to plan the parametric process that will be incorporated in the design solution. Although the use of graphic scripting does not require specific programing knowledge, it still requires understanding of programming logic. Nonetheless, it is considered a more accessible language for architects.

In addition to the requirements described above, it is necessary to build mathematical thinking, organization, and process characterization skills. It is understood that technologies available for the development of architectural design solutions are in constant evolution. The use of these technologies for the advancement of architectural practice is a critical component for building performance improvement. Integrated modeling and architectural design approaches, as well as the incorporation of evaluation cycles to such processes are indispensable.

Future work will discuss the development of integrated, single interface, multi simulation parametric and algorithmic models, as well as academic exercises that incorporate 
the presented application in architectural studio settings. Professional practice transformation, in response to the current Brazilian architectural production scenario, necessarily requires a critical stand on architectural curricula and demands on architectural practice. It is essential to open discussions on how these considerations will be incorporated in the contemporary education of our architects and urban planners.

\section{References}

Celani, g.; Vaz, c. Cad scripting and visual programming languages for implementing computational design concepts: a comparison from a pedagogical point of view. international journal of architectural computing, v. 10, n. 1, p. 121-138, 2012. Eastman, C. et al. Bim case studies. Bim handbook: a guide to building information modeling for owners, managers, designers, engineers, and contractors, p. 319-465, 2008

Givoni, b. Comfort, climate analysis and building design guidelines. energy and buildings, v. 18, n. 1, p. 11-23, 1992.

Leach, $\mathrm{N}$. the limits of urban simulation: an interview with manuel delanda. architectural design, v. 79, n. 4, p. 50-55, 2009.

. Parametrics explained. Next generation building, v. 1, n. 1, p. 33-42, 2014.

Motta, E. Reusable components for knowledge modelling: case studies in parametric design problem solving. ios press,1999.

Oxman,N. Material based design computation. MIT. 2010.

Turrin, M.; Von Buelow, P.; Stouffs, r. Design explorations of performance driven geometry in architectural design using parametric modeling and genetic algorithms. Advanced engineering informatics, v. 25, n. 4, p. 656-675, 10// 2011.

Woodbury, R. Elements of parametric design. Ed. Routledge, 2010. 